\title{
Cold Attractive Spin Polarized Fermi Lattice Gases and the Doped Positive U Hubbard Model
}

\author{
Adriana Moreo ${ }^{1}$ and D. J. Scalapino ${ }^{2}$ \\ ${ }^{1}$ Department of Physics and Astronomy, University of Tennessee, Knoxville, TN 37966-1200 and \\ Oak Ridge National Laboratory,Oak Ridge, TN 37831-6032,USA, and \\ ${ }^{2}$ Department of Physics, University of California, Santa Barbara, CA 93106-9530 USA
}

(Dated: August 9, 2018)

\begin{abstract}
Experiments on polarized fermion gases performed by trapping ultracold atoms in optical lattices, allow the study of an attractive Hubbard model for which the strength of the on site interaction is tuned by means of a Feshbach resonance. Using a well-known particle-hole transformation we discuss how results obtained for this system can be reinterpreted in the context of a doped repulsive Hubbard model. In particular we show that the Fulde-Ferrell-Larkin-Ovchinnikov (FFLO) state corresponds to the striped state of the two-dimensional doped positive U Hubbard model. We then use the results of numerical studies of the striped state to relate the periodicity of the FFLO state to the spin polarization. We also comment on the relationship of the $d_{x^{2}-y^{2}}$ superconducting phase of the doped $2 \mathrm{D}$ repulsive Hubbard model to a d-wave spin density wave state for the attractive case.

PACS numbers: 71.10.Fd, 03.75.Ss, 74.25.Ha
\end{abstract}

Using standing wave laser light fields and optical Feshbach resonances, ultracold atomic gases can be used to realize a variety of Hubbard like models,$\frac{1,2}{2}$ Technically if one wants a strong interaction, it appears easier to create an ultracold fermi gas (e.g., $\left.{ }^{6} \mathrm{Li}\right)$ with an attractive interaction and experiments on polarized fermion gases with attractive interactions have been carried out to look for exotic superfluid states $\frac{3,4,5,6}{3}$

One state of particular interest is the FFLO state which arises from pairing across the spin-split Fermi surface of a spin polarized system .7 .8 In this state the Cooper pairs have a finite center of mass momentum leading to spatial oscillations of the pair field order parameter and the spin polarization. Here, making use of density-matrix-renormalization-group (DMRG) results 9.10 for the doped, positive U Hubbard model and a well-known particle-hole transformation, $11,12,13,14$ we discuss the properties of the FFLO state for the case of a half-filled spin polarized negative U Hubbard model on a two-dimensional lattice. We also comment on the relationship of this to the question of whether the doped positive U Hubbard model may have a $d_{x^{2}-y^{2}}$ superconducting ground state.

The Hamiltonian for a half-filled negative U Hubbard model in an external Zeeman field, $H$, can be written as

$$
\begin{gathered}
\mathcal{H}=-t \sum_{\langle i j\rangle s}\left(c_{i s}^{\dagger} c_{j s}+c_{j s}^{\dagger} c_{i s}\right)+\frac{U}{2} \sum_{i}\left(n_{i \uparrow}-n_{i \downarrow}\right)^{2} \\
-h \sum_{i}\left(n_{i \uparrow}-n_{i \downarrow}\right) .
\end{gathered}
$$

Here $t$ is a nearest neighbor one-electron hopping, $U$ is positive so the onsite interaction is $-U n_{i \uparrow} n_{i \downarrow}$ and $h=$ $g \mu_{0} H / 2$. The operator $c_{i s}^{\dagger}$ creates a fermion with spin $s$ on site $i$, the sum $\langle i j\rangle$ is over nearest neighbor sites of a square lattice and $n_{i s}=c_{i s}^{\dagger} c_{i s}$ is the number operator. In the case of the ultracold gases, a spin polarization $p=\left(N_{\uparrow}-N_{\downarrow}\right) / N$ is achieved by loading more atoms in the "up" pseudo-spin hyperfine state than in the "down" state.

In the mean-field FFLO state, the Cooper pairs have a finite center of mass momentum which, for a two dimensional tight binding band structure, lays along the $(1,0)$ or the $(1,1)$ direction depending upon the ratio of $|U| / t$. For the case in which it lays along the $(1,0)$ direction, the order parameter exhibits a one-dimensional oscillation along the $x$ direction

$$
\Delta\left(\ell_{x}\right)=\operatorname{Re}\left\langle c_{\ell \uparrow} c_{\ell \downarrow}\right\rangle=-\Delta_{0} \cos \left(q_{x} \ell_{x}\right) .
$$

Here we have chosen the phase so that $\Delta(0)$ is negative. The spin polarization also varies in space with

$$
n_{s}\left(\ell_{x}\right)=\left\langle n_{\ell \uparrow}-n_{\ell \downarrow}\right\rangle=p-m_{0} \cos \left(2 q_{x} \ell_{x}\right) .
$$

Here $\cos \left(q_{x} \ell_{x}\right)$ and $\cos \left(2 q_{x} \ell_{x}\right)$ give the leading weak coupling harmonic behavior. The spatial variation of $\Delta\left(\ell_{x}\right)$ and $n_{s}\left(\ell_{x}\right)$ are schematically illustrated in Fig. 1. At stronger coupling higher harmonics can enter giving a more localized behavior.

Since the possibility of an FFLO state was originally proposed,,$\frac{7.8}{t}$ there has been great interest in determining whether it exists and exploring its properties. In particular, with the possibility of observing such a state in ultracold atomic gases, there have been a number of theoretical calculations $\frac{15,16,17,18,19,20,21}{1}$ These calculations have been based upon a mean field description. Here we are interested in the case of a two-dimensional half-filled lattice gas where it is important to treat strong coupling effects. One would like to know if the FFLO state survives beyond the mean field approximation, whether the 
stripes run vertically (or horizontally) as in Eqs. (2) and (31) or diagonally, and whether the periodicity of the spatial variation which determines $q_{x}$ remains at its mean field value.

The Hamiltonian for the negative U Hubbard model in a Zeeman field can be transformed under a unitary transformation to a doped positive U Hubbard model. This transformation, introduced by Emery $\stackrel{11}{\rightleftarrows}$ has been a staple in quantum Monte Carlo work where it was used to show that the fermion sign problem is absent for the halffilled Hubbard model with a near-neighbor hopping. $\underline{12}^{2}$ It has also been used, as we will review, to provide a map between the half-filled negative U Hubbard model in a Zeeman field and the doped positive U Hubbard model $\stackrel{12,13,14,22}{1,2}$ Under this transformation we will see that the FFLO state becomes the familiar striped, charge density, and $\pi$-phase shifted antiferromagnetic state of the doped positive U Hubbard model.

For a unitary transformation in which

$$
c_{\ell \uparrow} \rightarrow(-1)^{\ell_{x}+\ell_{y}} d_{\ell \uparrow}^{\dagger} \quad c_{\ell \downarrow} \rightarrow d_{\ell \downarrow}
$$

the Hamiltonian given in Eq. (11) becomes

$$
\begin{gathered}
\mathcal{H}=-t \sum_{\langle i j\rangle s}\left(d_{i s}^{\dagger} d_{j s}+d_{j s}^{\dagger} d_{i s}\right)+\frac{U}{2} \sum\left(n_{i \uparrow}+n_{i \downarrow}-1\right)^{2} \\
-\mu \sum_{i}\left(n_{i \uparrow}+n_{i \downarrow}\right)
\end{gathered}
$$

with $\mu=-h$. That is, the half-filled negative U Hubbard model with spin polarization $p$ is mapped into a positive $\mathrm{U}$ Hubbard model with a site filling $\langle n\rangle=1-p$. Under this transformation Eq. (2) becomes

$$
(-1)^{\ell_{x}+\ell_{y}}\left\langle M_{x}\left(\ell_{x}, \ell_{y}\right)\right\rangle=-\Delta_{0} \cos q_{x} \ell_{x}
$$

with $M_{x}(\ell)=\left(d_{\ell \uparrow}^{\dagger} d_{\ell \downarrow}+d_{\ell \downarrow}^{\dagger} d_{\ell \uparrow}\right) / 2$. The system has rotational symmetry so that Eq. (6) implies that the staggered spin order is modulated by $\cos q_{x} \ell_{x}$.

$$
\begin{aligned}
m_{\text {stag }}\left(\ell_{x}\right) & =\frac{(-1)^{\ell_{x}+\ell_{y}}}{2}\left\langle n_{\uparrow}\left(\ell_{x}, \ell_{y}\right)-n_{\downarrow}\left(\ell_{x}, \ell_{y}\right)\right\rangle \\
& =-\Delta_{0} \cos q_{x} \ell_{x} .
\end{aligned}
$$

Similarly, the spin polarization, Eq. (3), transforms to the hole density giving

$$
h\left(\ell_{x}\right)=1-\left\langle n\left(\ell_{x}, \ell_{y}\right)\right\rangle=p-m_{0} \cos \left(2 q_{x} \ell_{x}\right) .
$$

Thus the FFLO state maps to the striped state of the doped positive U Hubbard model in which charged stripes separate $\pi$-phase shifted antiferromagnetic regions.
Mean field studies $13,23,24,25,26,27$ of the doped, twodimensional positive $\mathrm{U}$ Hubbard model with a nearestneighbor hopping find that the domain-walls run along the $x$ or $y$-directions for $|U| / t \lesssim 3.6$. For values of $3.6 \lesssim|U| / t \lesssim 8$ the domain-walls run diagonally and for $|U| / t$ greater than 8 the domain-walls are found to be unstable with respect to the formation of magnetic polarons $\stackrel{28}{=}$ The mean field domain walls are found to contain one hole per site along the wall and one says that the domain wall is filled with holes. This means that the spacing of the vertical (or horizontal) charged domain walls is equal to $p^{-1}$, giving the mean field result

$$
q_{x}=\pi p .
$$

for such walls. A schematic illustration of the mean field results for $p=0.125$ is shown in Fig. 1h.

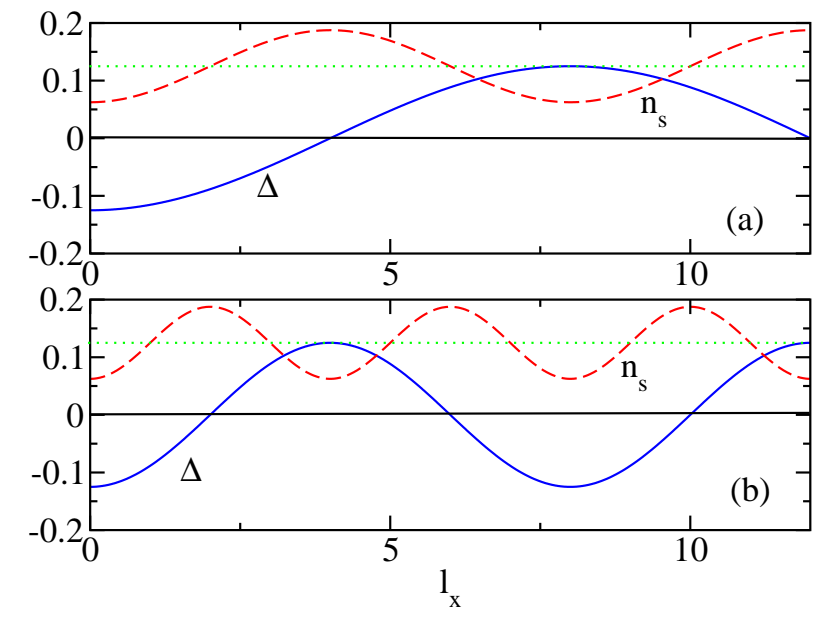

FIG. 1: (Color on line) Schematic results for the spatial variation of the $s$-wave order parameter $\Delta\left(\ell_{x}\right)$ (solid) and the spin polarization $n_{s}\left(\ell_{x}\right)$ (dashed) in an FFLO state with $p=0.125$. (a) The mean field result with a period for $\Delta\left(\ell_{x}\right)$ of $2 / p=16$ and (b) with a period $1 / p=8$ expected from DMRG calculations on long stripes.

Now these are mean field results and at larger values of $\mathrm{U}$ one is dealing with a strongly correlated system. Thus one might wonder whether the FFLO state remains the ground state. This is a question that has also played a central role in the high $T_{c}$ cuprate problem. There one would like to know if the ground state of the doped Hubbard model is striped or possibly a $d_{x^{2}-y^{2}}$ superconductor, and what is the nature of the interplay between stripes and pairing. ${ }^{29}$ Present DMRG calculations on 6-leg Hubbard ladders find a striped ground state. ${ }^{9.10}$, while dynamic cluster Monte Carlo calculations ${ }^{30}$ on periodic lattices find evidence of a $d_{x^{2}-y^{2}}$ superconducting state. Whether the difference of lattice aspect ratios and boundary conditions or subtle numerical biases are responsible for this disagreement is not known. It does, however, appear that these two states are very close in energy. Here we will use the DMRG results for the striped 
state to discuss the nature of the FFLO state and then conclude by noting what would happen for the $d_{x^{2}-y^{2}}$ state.

The DMRG calculations have been carried out on 6leg doped positive U Hubbard ladders. Periodic boundary conditions have been imposed in the 6-leg direction and open boundary conditions are used in the long $x$ direction. In Ref. 10, the results have been extrapolated to infinite length ladders. These numerical results find that the ground state exhibits the striped structure shown in Fig. 2 for a $6 \times 21$ ladder with $U / t=12$ and 12 holes corresponding to a doping $p=2 / 21$. The figure shows the hole density

$$
h\left(\ell_{x}\right)=\frac{1}{6} \sum_{\ell_{y}=1}^{6}\left(1-\left\langle n\left(\ell_{x}, \ell_{y}\right)\right\rangle\right)
$$

and the staggered spin density

$$
m_{\text {stag }}\left(\ell_{x}\right)=\frac{1}{6} \sum_{\ell_{y}=1}^{6}(-1)^{\ell_{x}+\ell_{y}}\left\langle n_{\uparrow}\left(\ell_{x}, \ell_{y}\right)-n_{\downarrow}\left(\ell_{x}, \ell_{y}\right)\right\rangle,
$$

versus $\ell_{x}$. For this doping, extrapolated DMRG results 10 show that the ground state is striped for $U / t \gtrsim 3$. For a 6 -leg tube, the stripes are found to contain 4 holes (2/3 filled stripes) so that the charge stripe spacing is $2 /(3 p)=7$ with $\langle n\rangle=1-p$.

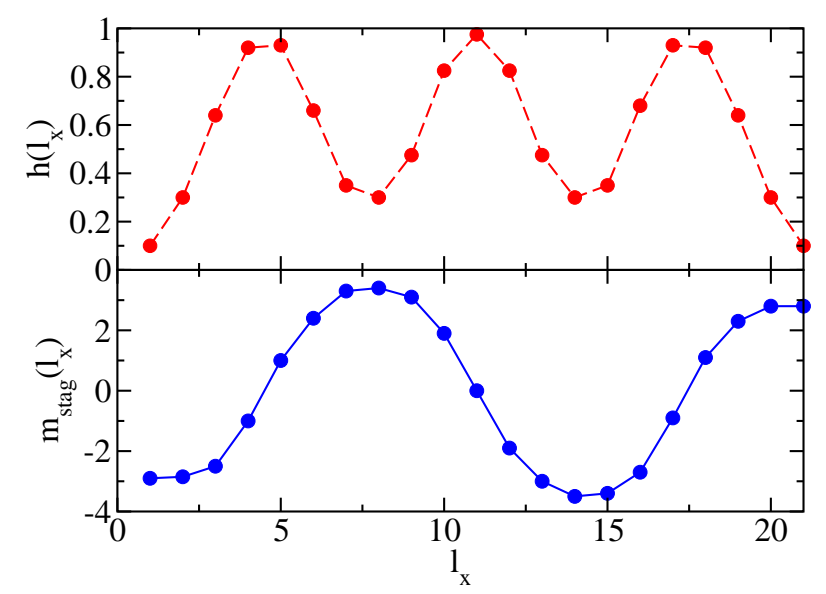

FIG. 2: (Color online). This figure shows DMRG results (G. Hager et al. $\left.{ }^{10}\right)$ for the hole $h\left(\ell_{x}\right)$ (dashed red) and the staggered spin $m_{\text {stag }}\left(\ell_{x}\right)$ (solid blue) densities along the leg direction for a $21 \times 6$ Hubbard ladder with 12 holes and $U / t=$ 12. As discussed in the text, $h\left(\ell_{x}\right)$ corresponds to the spin polarization $n_{s}\left(\ell_{x}\right)$ and $m_{\text {stag }}\left(\ell_{x}\right)$ corresponds to the $s$-wave pairfield order parameter $\Delta\left(\ell_{x}\right)$ of the FFLO state.

Calculations on an 8-leg $t-J$ ladder find that the stripes are half-filled and there is evidence that this is the preferred filling. $\underline{31}$ These calculations show that the holes that make up the stripe exhibit short range pairing correlations ${ }^{32}$ in which the sign of the singlet pair field associated with two sites connected by a hopping perpendicular to the stripe is opposite to that of the case in which the hopping is parallel to the stripe. These d-wave like pairing correlations of the holes in the stripe are not taken into account in the mean field calculations which find filled stripes. For half-filled stripes, the spacing between the charged stripes is $1 / 2 p$ so that the numerical calculations on the longer stripes imply that $q_{x}$ for the FFLO state will be twice that found in the mean field calculations.

$$
q_{x}=2 \pi p .
$$

In this case, the FFLO state will have half the spatial period of the mean field solution, as illustration in Fig. [1b.

Here we have made use of DMRG calculations which were carried out on systems with periodic boundary conditions in one direction and open boundary conditions in the other. In cold atoms experiments, one is generally dealing with an underlying optical lattice in a slowly varying trap potential which acts as a spatially dependent chemical potential. This leads to soft boundaries and the simultaneous coexistence of spatially separated phases. The details of this depend upon the total filling as well as the curvature of the confining trap potential. ${ }^{33}$ In principle, the DMRG method is well suited to treating such soft boundaries but here we have focused our discussion on the idealized case of a two-dimensional lattice which has one fermion per site with an attractive Feshbach tuned interaction and a spin polarization $p$. We have shown that the FFLO state of a $2 \mathrm{D}$ half-filled attractive spin polarized Hubbard model is a unitary transform of the striped state of the doped repulsive Hubbard model. Thus an experimental observation of the FFLO state in the above mentioned cold atom set-up would imply that the 2D doped repulsive Hubbard model is striped. Note that we have not shown that the striped state is the ground state of the two-dimensional doped repulsive U Hubbard model. This remains an open question. We have simply shown that if the striped state were the ground state then this would imply that the FFLO state would be found in the optical lattice experiments and that its periodicity would be given by Eq. (12). We believe that the factor of two increase in $q_{x}$ represents an important change from the mean field result and reflects the tendency towards $d_{x^{2}-y^{2}}$ pairing, ${ }^{32}$ Recent experiments also suggest that local pairing correlations are present on the cuprate stripes $\underline{\underline{34}}$

Finding evidence for an FFLO state in a cold attractive spin polarized fermi lattice gas would provide evidence that the low temperature phase of the doped positive U Hubbard model is striped. If on the other hand, the $d_{x^{2}-y^{2}}$ superconducting state describes the low temperature phase of the repulsive Hubbard model, then the transformation, Eq. (4), tells us that the cold atom system will exhibit a "d-spin density wave" ground state characterized by an order parameter 


$$
\sum_{k}\left(\cos k_{x}-\cos k_{y}\right)\left\langle c_{k+Q \uparrow}^{\dagger} c_{k \downarrow}\right\rangle
$$

with $Q=(\pi, \pi)$. Thus experiments on half-filled, spin polarized cold fermi lattice gases with attractive on site interactions and the search for the FFLO state have important implications for the properties of the doped repulsive U Hubbard model. Likewise, our present understanding of the repulsive Hubbard model can provide information relevant to experiments on the spin polarized attractive fermi gas.

\section{Acknowledgements}

We would like to thank T. Esslinger, E. Mueller, and M. Holland for helpful discussions regarding the recent experimental work on cold fermion gases and L. Balents, S.A. Kivelson, and I. Affleck for useful discussions regarding the FFLO state. We would also like to thank G. Hager, G. Wellein, E. Jeckelmann and H. Fehske for providing the data for Figure 2. A.M. is supported by NSF under grants DMR-0443144 and DMR-0454504, and partial support under Contract DE-AC05-00OR22725 with UT-Battelle, LLC. D.J.S. would like to acknowledge the Center for Nanophase Material Science at Oak Ridge National Laboratory for support.
1 D. Jaksch et al., Phys. Rev. Lett.81, 3108 (1998).

2 M. Holland et al., Phys. Rev. Lett.87, 120406 (2001).

3 M. Köhl et al., Phys. Rev. Lett.94, 80403 (2005).

4 M.W. Zwierlein et al., Science 311, 492 (2006).

${ }^{5}$ G.B. Partridge et al., Science 311, 503 (2006).

6 Y. Shin et al., cond-mat/0606432,

7 P. Fulde and R.A. Ferrell, Phys. Rev.135, A550 (1964).

8 A.I. Larkin and Y.N. Ovchinnikov, Sov. Phys. JETP 20, $762(1965)$.

9 S.R. White and D.J. Scalapino, Phys. Rev. Lett. 91, 136403 (2003).

10 G. Hager et al., Phys. Rev. B71, 75108 (2005).

11 V.J. Emery, Phys. Rev. B14, 2989 (1976).

12 J.E. Hirsch, Phys. Rev. B31, 4403 (1985).

13 H.J. Schulz, J. Phys. France 50, 2833 (1989).

14 M.I. Salkola and J.R. Schrieffer, Phys.Rev.B57,14433 (1998).

15 J. Carlson and S. Reddy, Phys. Rev. Lett. 95, 60401 (2005).

16 C.-H. Pao, S.-T. Wu and S.-K. Yip, Phys. Rev. B73, 132506 (2006).

17 D.E. Sheehy and L. Radzihovsky, Phys. Rev. Lett. 96, 60401 (2006).

18 K. Machida, T. Mizushima and M. Ichioka, Phys. Rev. Lett. 97, 120407 (2006).

19 D.E. Sheey and L. Radzihovsky, cond-mat/0607803, 0608172 .
${ }^{20}$ L. He, M. Jin and P. Zhuang, cond-mat/0606322.

${ }^{21}$ Y. He, C.-C. Chien, Q. Chen and K. Levin, cond-mat/0610274.

22 R. Scalettar et al., Phys. Rev. Lett. 62, 1407 (1989).

23 J. Zaanen and O. Gunnarsson, Phys. Rev.B40, 7391 (1989).

24 D. Poilblanc and T.M. Rice, Phys. Rev. B39, 9749 (1989).

25 T. Giamarchi and C. Lhuillier, Phys. Rev. B42, 10641 (1990).

26 M. Inui and P.B. Littlewood, Phys. Rev.B44, 4415 (1991).

27 Q. Wang, H.-Y. Chen, C.-R. Hu and C.S. Ting, Phys. Rev. Lett. 96, 117006 (2006).

28 Here we have quoted results that Inui and Littlewood ${ }^{26}$ obtained from a Hartree-Fock study of a two-dimensional Hubbard model with a near neighbor hopping.

29 S.A.Kivelson and E. Fradkin, cond-mat/0507459

30 T.A. Maier et al., Phys. Rev. Lett. 95, 237001 (2005).

31 S.R. White and D.J. Scalapino, Phys. Rev. Lett. 91, 3227 (1998).

32 S.R. White and D.J. Scalapino, "Why do stripes form in doped antiferromagnets and what is their relationship to superconductivity?", cond-mat/0006071.

33 M. Rigol and A. Muramatsu, Phys. Rev.A69, 53612 (2004).

34 T. Valla et al., Sciencexpress Report, 16 November 2006, page 1 . 